\title{
Resting-State Functional Connectivity Indexes Reading Competence in Children and Adults
}

\author{
Maki S. Koyama, ${ }^{1}$ Adriana Di Martino, ${ }^{1}$ Xi-Nian Zuo, ${ }^{1,2}$ Clare Kelly, ${ }^{1}$ Maarten Mennes, ${ }^{1}$ Devika R. Jutagir, ${ }^{1}$ \\ F. Xavier Castellanos, ${ }^{1,3}$ and Michael P. Milham ${ }^{1,3}$ \\ ${ }_{1}^{1}$ hyllis Green and Randolph Cowen Institute for Pediatric Neuroscience at the New York University Child Study Center, New York, New York 10016, \\ ${ }^{2}$ Laboratory for Functional Connectome and Development, Key Laboratory of Behavioral Science, Institute of Psychology, Chinese Academy of Sciences, \\ Beijing, 100101, China, and ${ }^{3}$ Nathan Kline Institute for Psychiatric Research, Orangeburg, New York 10962
}

Task-based neuroimaging studies face the challenge of developing tasks capable of equivalently probing reading networks across different age groups. Resting-state fMRI, which requires no specific task, circumvents these difficulties. Here, in 25 children (8-14 years) and 25 adults (21-46 years), we examined the extent to which individual differences in reading competence can be related to resting-state functional connectivity (RSFC) of regions implicated in reading. In both age groups, reading standard scores correlated positively with RSFC between the left precentral gyrus and other motor regions, and between Broca's and Wernicke's areas. This suggests that, regardless of age group, stronger coupling among motor regions, as well as between language/speech regions, subserves better reading, presumably reflecting automatized articulation. We also observed divergent RSFC- behavior relationships in children and adults, particularly those anchored in the left fusiform gyrus (FFG) (the visual word form area). In adults, but not children, better reading performance was associated with stronger positive correlations between FFG and phonology-related regions (Broca's area and the left inferior parietal lobule), and with stronger negative relationships between FFG and regions of the "task-negative" default network. These results suggest that both positive RSFC (functional coupling) between reading regions and negative RSFC (functional segregation) between a reading region and default network regions are important for automatized reading, characteristic of adult readers. Together, our taskindependent RSFC findings highlight the importance of appreciating developmental changes in the neural correlates of reading competence, and suggest that RSFC may serve to facilitate the identification of reading disorders in different age groups.

\section{Introduction}

Reading is a relatively recent cultural invention. As such, the human brain lacks functional networks innately specialized for reading. Instead, intensive learning over childhood and adolescence contributes to the development of reading networks. Such learning-induced cortical plasticity is difficult to capture with traditional task-based neuroimaging studies, primarily due to the challenge of developing tasks capable of equivalently probing reading networks across different age groups (Schlaggar and Church, 2009). Some developmental imaging studies have tackled this problem by restricting the age range under investigation (Bitan et al., 2007; Booth et al., 2008) or by using implicit reading tasks (e.g., visual feature detection of letter strings) that can be performed equivalently across different age groups (Turkeltaub et al., 2003). However, as previously noted (Church et al., 2010), matching task performance between age groups does not neces-

\footnotetext{
Received Sept. 16, 2010; revised March 9, 2011; accepted April 14, 2011.

Author contributions: C.K. and M.P.M. designed research; A.D.M., F.X.C., and M.P.M. performed research;X.-N.Z. and M.M. contributed unpublished reagents/analytic tools; M.S.K. analyzed data; M.S.K. and D.R.J. wrote the paper.

This research was partially supported by grants from NIMH [R01MH081218 (F.X.C.), K23MH087770 (A.D.M.)], the Stavros Niarchos Foundation (F.X.C.), and the Leon Levy Foundation (M.P.M., A.D.M.).

Correspondence should be addressed to either Dr. Maki S. Koyama or Dr. Michael P. Milham, Phyllis Green and Randolph Cowen Institute for Pediatric Neuroscience at the New York University Child Study Center, New York, NY 10016. E-mail:makisophiakoyama@gmail.com or michael.milham@nyumc.org.

DOI:10.1523/JNEUROSCI.4865-10.2011

Copyright $\odot 2011$ the authors $\quad 0270-6474 / 11 / 318617-08 \$ 15.00 / 0$
}

sarily constrain the cognitive strategies or neural networks used during the task. Thus, although these approaches have been of unquestionable value, their ability to account for the development of neural mechanisms underlying reading may be limited.

One way to bypass these difficulties is to investigate restingstate functional connectivity (RSFC), which requires no reading task or word stimuli. This task-independent approach detects inter-regional correlations among spontaneous low-frequency $(<0.1 \mathrm{~Hz})$ fluctuations in the fMRI signal (Biswal et al., 1995). This method has successfully characterized myriad functional brain networks that resemble patterns of activation observed during task performance (Toro et al., 2008; Smith et al., 2009). Relevant to the current study, recent work has demonstrated that RSFC can map reading networks previously identified in taskbased imaging studies (Hampson et al., 2006; Koyama et al., 2010). However, these studies focused exclusively on experienced adult readers, leaving the organization of reading networks in children (i.e., less experienced readers) relatively unexplored.

Here, we examined how RSFC within known reading networks varies in association with reading competence in children and adults. Studies investigating RSFC-behavior relationships have shown that individual differences in performance (Wang et al., 2010) and traits (Di Martino et al., 2009; Cox et al., 2010) are associated with individual differences in the strength of RSFC. Probing 11 seeds [regions of interest (ROIs)] derived from the reading literature (Bolger et al., 2005; Houdé et al., 2010; Koyama 
Table 1. Summary of demographic and cognitive measures for each age group

\begin{tabular}{llll}
\hline & $\begin{array}{l}\text { Children } \\
(n=25)\end{array}$ & $\begin{array}{l}\text { Adults } \\
(n=25)\end{array}$ & $\begin{array}{l}\text { Group } \\
\text { differences }\end{array}$ \\
\hline Sex (M/F) & $13 \mathrm{M} / 12 \mathrm{~F}$ & $10 \mathrm{M} / 15 \mathrm{~F}$ & N.S. \\
Age (years) & $11.7 \pm 1.6$ & $31.6 \pm 8.2$ & $p<0.001$ \\
Age range (years) & $8-14$ & $20-46$ & \\
Word reading raw score & $111.6 \pm 11.9$ & $127.1 \pm 4.1$ & $p<0.001$ \\
Word reading standard score & $110.6 \pm 8.9$ & $106.2 \pm 7.0$ & N.S. \\
Full-scale IQ & $111.9 \pm 11.5$ & $110.8 \pm 10.2$ & N.S. \\
\hline
\end{tabular}

M, Males; F, females.

et al., 2010), we aimed to identify: (1) functional connections in which the relationship between RSFC and reading competence was common between the two age groups, and (2) functional connections in which the relationship between RSFC and reading competence was divergent between the two age groups. These aims were based on converging evidence that (1) children and adults recruit largely similar, but not identical, neural networks while reading (Gaillard et al., 2003; Church et al., 2008; Turkeltaub et al., 2008), yet (2) developmental changes in functional networks continue beyond childhood (Fair et al., 2009; Kelly et al., 2009; Supekar et al., 2009; Dosenbach et al., 2010; Zuo et al., 2010).

\section{Materials and Methods \\ Participants}

Fifty right-handed native English speakers ( 25 children, aged 8 - 14 years, mean age $=12 \pm 2$ years, 13 males; 25 adults, aged $20-46$ years, mean age $=32 \pm 8$ years, 10 males) participated. No participant had psychiatric, cognitive, or reading disorders, as confirmed by semistructured psychiatric interviews and abbreviated cognitive testing. Absence of axis I psychiatric disorders was based on the Schedule for Affective Disorders and Schizophrenia for School-Age Children-Present and Lifetime Version (KSADS-PL) (Kaufman et al., 1996), which was administered to parents and their children ( $<18$ years old) separately. The Structured Clinical Interview for DSM-IV-TR Axis I Disorders, Research Version, Non-patient Edition (SCID-I/NP) (First et al., 2002) and the Adult ADHD Clinical Diagnostic Scale (ACDS) V.1.2 (Adler and Spencer, 2004) were administered to adults ( $>18$ years old). To evaluate intellectual and reading abilities in both age groups, we administered the four subtests of the Wechsler Abbreviated Scale of Intelligence (WASI) (Wechsler, 1999) and the Word Reading subtest from the Wechsler Individual Achievement Test-Second Edition (WIAT-II) (Wechsler, 2001), respectively. Table 1 provides demographic and cognitive measures for each age group.

As shown in Figure 1A, children's reading performance measured by word reading raw scores reached the adult level plateau at 15 years of age. This is consistent with the expected development of word reading (Wechsler, 2001). A similar developmental pattern was also found in the relationship between verbal IQ raw scores and age (Fig. $1 B$ ). As shown in Figure $1(C$ and $D)$, we matched the two age groups on age-normalized standard scores of word reading and full-scale IQ. This ensured that potential group differences could not be attributed to differences in agenormalized (or age-appropriate) abilities of reading, or of general intelligence. This study was approved by the institutional review boards of the New York University School of Medicine and New York University. Before participation, written informed consent was obtained from all participants and their legal guardians (in the case of children).

\section{MRI data acquisition}

MRI data were collected on a Siemens Allegra 3.0 T scanner at the New York University Center for Brain Imaging. We collected resting-state fMRI data in an oblique plane using a customized multi-echoplanar imaging (EPI) sequence to mitigate the effects of susceptibility artifacts in medial temporal and ventromedial regions (Fleysher et al., 2005): 180 whole-brain volumes; $\mathrm{TR}=2000 \mathrm{~ms}$; $\mathrm{TE}=30 \mathrm{~ms}$; flip angle $=90^{\circ} ; 33$
Word Reading
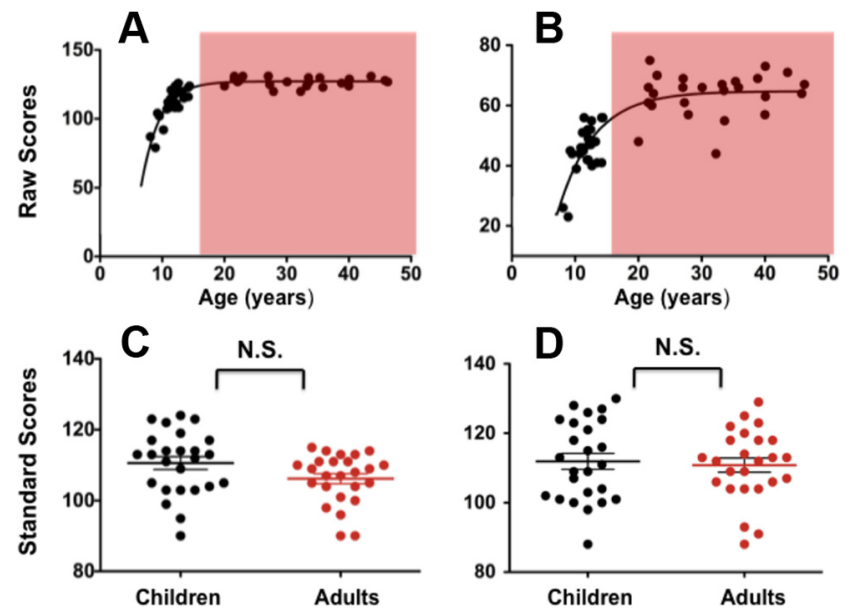

Figure 1. Raw and standard scores of word reading (WIAT) and verbal IQ (WASI). $\boldsymbol{A}, \boldsymbol{B}$, Scatter plots depict the relationship between age and raw scores of $(\boldsymbol{A})$ word reading and $(\boldsymbol{B})$ verbal IQ. Individuals $>20$ years (adults) are highlighted by the red shadow. $C, D$, There are no differences between the child and adult groups in standard scores of $(\boldsymbol{C})$ word reading and $(\boldsymbol{D})$ verbal IQ.

contiguous $4 \mathrm{~mm}$ slices; matrix $=80 \times 64$; acquisition voxel size $=3 \times$ $3 \times 4 \mathrm{~mm}$. This sequence uses the dead time that precedes the readout in EPI sequences with normal TEs $(\sim 30 \mathrm{~ms})$ to collect additional images at several echo times. The first is a low-resolution, fully sampled image (matrix size $80 \times 12$ ) with an echo time of $7.32 \mathrm{~ms}$; the second is a partial Fourier (5/8) image at full resolution with an echo time of $12.12 \mathrm{~ms}$ and with reversed phase-encoding gradients; and the third is a conventional fully sampled image with echo time of $32.92 \mathrm{~ms}$. This scheme allows for optimal detection of signal from fast-decay (short $\mathrm{T} 2^{*}$ ) regions and slower-decay (long $\mathrm{T}^{*}$ ) regions. Collecting one of the images with reversed phase-encoding gradients allows correction for susceptibility artifacts even in the presence of substantial subject motion. For spatial normalization and localization, we also acquired a high-resolution T1weighted magnetization-prepared gradient echo sequence $(\mathrm{TR}=2530$ $\mathrm{ms}$; $\mathrm{TE}=3.25 \mathrm{~ms}$; $\mathrm{TI}=1100 \mathrm{~ms}$; flip angle $=7^{\circ}$; 192 slices; field of view $=100 \mathrm{~mm})$.

\section{Data preprocessing}

For each participant, image preprocessing was performed using a combination of Analysis of Functional NeuroImages (AFNI) (Cox, 1996) and FMRIB Software Library (FSL) (http://www.fmrib.ox.ac.uk/fsl/). Scripts containing data-processing steps similar to those used here have been released as part of the 1000 Functional Connectomes Project (Biswal et al., 2010) (http://www.nitrc.org/projects/fcon_1000). Preprocessing comprised: (1) discarding the first 4 EPI volumes from each scan to allow for signal equilibration, (2) slice timing correction, (3) 3D motion correction, (4) time series despiking (detection and reduction of extreme time series outliers), (5) spatial smoothing (6 mm FWHM Gaussian kernel), (6) four-dimensional (4D) mean-based intensity normalization, (7) bandpass temporal filtering $(0.009-0.1 \mathrm{~Hz})$, and (8) removal of linear and quadratic trends. Registration of each participant's high-resolution anatomical image to standard $2 \mathrm{~mm}^{3} \mathrm{MNI}$ space was accomplished in two steps (Andersson et al., 2007). First, a $12 \mathrm{df}$ linear affine transformation was computed using FLIRT (Jenkinson and Smith, 2001; Jenkinson et al., 2002). Subsequently, the registration was refined using FNIRT (Andersson et al., 2007). To control for potential effects of physiological processes and motion, each participant's preprocessed data were regressed on nine nuisance covariates (signals from white matter, cerebrospinal fluid, the global signal, and six motion parameters) (Kelly et al., 2008). The resultant $4 \mathrm{D}$ residual time series were used for subsequent participant-level analyses. 


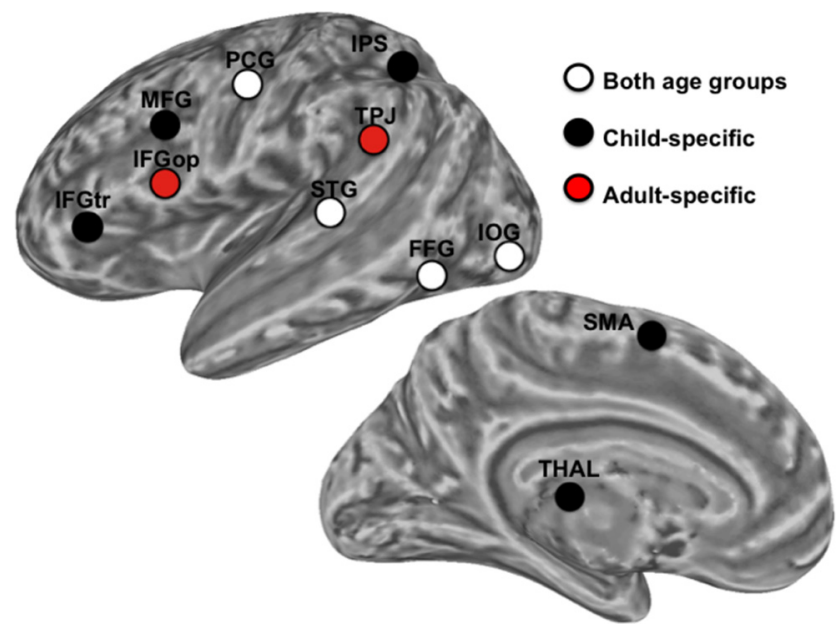

Figure 2. Seed ROls. Seeds are presented as overlaid circles; white circles indicate the 4 ROIs reported in both child-based and adult-based meta-analyses of reading (Both age groups), black circles indicate the 5 ROIs reported only in the child meta-analysis (Child-specific), and red circles indicate the 2 ROls reported only in the adult meta-analysis (Adult-specific). See Table 2 for abbreviations of seed ROls.

\section{Seed ROIs}

We examined RSFC associated with 11 seed regions derived from metaanalyses of reading in children (Houdé et al., 2010) and adults (Bolger et al., 2005). Six of these seeds, based on the meta-analysis of reading in adults, were previously examined in a study validating RSFC as a probe of reading networks in healthy adults (Koyama et al., 2010). We examined the distances between each of the regions identified in the child-based meta-analysis and those reported in the adult meta-analysis to identify: (1) regions common to both child and adult meta-analyses: regions in the child meta-analysis that are within $15 \mathrm{~mm}$ of a region reported in the adult meta-analysis, (2) regions specific to the child meta-analysis: regions in the child meta-analysis that are beyond $15 \mathrm{~mm}$ of any region reported in the adult meta-analysis, and (3) regions specific to the adult meta-analysis. This approach identified: (1) four common regions [inferior occipital gyrus (IOG), posterior fusiform gyrus (FFG), posterior superior temporal gyrus (STG), dorsal precentral gyrus (PCG)], (2) five child-specific regions [intraparietal sulcus (IPS), supplementary motor area (SMA), inferior frontal gyrus pars triangularis (IFGtr), middle frontal gyrus (MFG), and thalamus (THAL)], and (3) two adult-specific regions [temporoparietal junction (TPJ), inferior frontal gyrus pars opercularis (IFGop)]. See Figure 2 and Table 2 for summary and region coordinates. Each of these regions was used as seed ROIs $\left(2 \mathrm{~mm}^{3}\right.$ standard space, radius $\left.=6 \mathrm{~mm}\right)$.

\section{Participant-level RSFC analyses}

For each seed, we extracted the mean time series from each participant's $4 \mathrm{D}$ residuals volume by averaging across all voxels in each seed in standard MNI152 $2 \mathrm{~mm}^{3}$ space. Using these mean time series, we performed a whole-brain correlation analysis for each participant and each seed using the AFNI program 3dfim + in each individual's native space. This analysis produced participant-level correlation maps of all voxels in the brain that were positively or negatively correlated with the seed's time series. Finally, these correlation maps were converted to $Z$-value maps using Fisher's $r$-to- $z$ transformation.

\section{Group-level RSFC analyses}

First, all participant-level $z$-transformed correlation maps were transformed to standard MNI152 $2 \mathrm{~mm}^{3}$ space by applying the previously computed transformation. Then, group-level analyses were performed using a mixed-effects ordinary least-squares model implemented in FSL. All analyses were corrected for multiple comparisons using Gaussian random field theory $(Z>2.3$, cluster-wise $p<0.05$, corrected).
Table 2. MNI coordinates of seed ROIs

\begin{tabular}{lrrr}
\hline & \multicolumn{3}{c}{ MNI coordinates } \\
\cline { 2 - 4 } Seed ROIs & $x$ & $y$ & $z$ \\
\hline Regions common to both child and adult meta-analyses & & & \\
IOG: Inferior occipital gyrus (posterior) & -25 & -87 & -10 \\
FFG: Fusiform gyrus (posterior) & -48 & -57 & -20 \\
STG: Superior temporal gyrus (posterior) & -53 & -31 & 9 \\
PCG: Precentral gyrus (dorsal) & -48 & -12 & 45 \\
Regions specific to the child meta-analysis & & & \\
IPS: Intraparietal sulcus (posterior) & -30 & -58 & 48 \\
SMA: Supplementary motor area & -4 & 10 & 58 \\
IFGtr: Inferior frontal gyrus (triangularis) & -48 & 32 & 6 \\
MFG: Middle frontal gyrus (ventral) & -44 & 10 & 30 \\
THAL: Thalamus & -10 & -14 & 8 \\
Regions specific to the adult meta-analysis & & & \\
TPJ: Temporoparietal junction & -59 & 45 & 15 \\
IFGop: Inferior frontal gyrus (opercularis) & -51 & 10 & 10 \\
\hline
\end{tabular}

"Regions common to both child and adult meta-analyses" corresponds to Rols that were reported in both childbased (Houdé et al., 2010) and adult-based (Bolger et al., 2005) meta-analyses of reading. "Regions specific to the child meta-analysis" and "Regions specific to the adult meta-analysis" are ROls that were reported only in the adult-based or child-based meta-analyses, respectively.

\section{Primary analyses}

Primary analyses aimed to delineate common and divergent relationships between RSFC strength and reading competence (reading standard scores) in the child and adult groups. Therefore, our group-level general linear model included separate covariates modeling reading standard scores for the child and adult groups (demeaned separately for each age group). As has been robustly demonstrated in the reading literature, full-scale IQ and reading standard scores were significantly correlated in both age groups (children, $r=0.72$, $\mathrm{df}=23, p=0.00005$; adults, $r=$ $0.64, \mathrm{df}=23, p=0.0006$ ). Thus, we modeled full-scale IQ scores as covariates of no interest to statistically remove any potential contributions of general intelligence to RSFC-reading competence relationships.

Common RSFC-reading competence relationships. Functional connections related to reading competence in both child and adult groups were identified using an $F$ test across the correlations between RSFC strength and reading competence in the child and adult groups. For each seed, the $F$ test produced a $Z$-statistic map of voxels whose RSFC with the seed varied significantly as a function of reading competence (i.e., exhibited a "main effect" of reading competence). All maps were corrected for multiple comparisons using cluster-level Gaussian random field correction $(Z>2.3, p<0.05$, corrected).

Importantly, following identification of regions via the $F$ test, we confirmed the presence of significant RSFC-behavior correlations $(p<$ $0.05)$ in each age group ( $R^{2}$ provided for all relationships). Additionally, we tested for the possibility that the RSFC-behavior correlations in the two groups may be similar in nature (i.e., positive or negative), though different in magnitude. Specifically, we compared the Fisher $z$-transformed RSFC-behavior correlations obtained in the two groups (child, adult) using the $Z$-statistic formula below:

$$
Z=\frac{z_{1}-z_{2}}{\sqrt{\frac{1}{n_{1}-3}+\frac{1}{n_{2}-3}}}
$$

In the equation, $z_{1}, n_{1}$ and $z_{2}, n_{2}$ correspond to the Fisher $z$-scoretransformed correlation coefficients and number of subjects in the child and adult groups, respectively. The null hypothesis is that no difference exists between the two groups with respect to the magnitude of the brainbehavior correlations. $Z$-statistics were transformed into $p$-values and the significance level was set at $p<0.05$.

Divergent RSFC-reading competence relationships. Functional connections, in which RSFC relationships with reading competence differed between the two age groups (i.e., age group specific), were identified through direct statistical comparisons of the RSFC-behavior relationships between the child and adult groups. These group-level contrasts 
produced $Z$-statistic maps showing voxels where correlations between the strength of RSFC with each seed and reading competence significantly differed between the child and adult groups (i.e., exhibited a group $\times$ reading competence interaction; $Z>2.3 ; p<0.05$, corrected).

Secondary analyses: ROI-based analyses

In addition to whole-brain voxelwise analyses, we performed ROI-based analyses in which we calculated RSFC between all possible pairings of the 11 reading seeds, creating an $11 \times 11$ correlation matrix for each participant (i.e., 55 unique pairwise correlations). Subsequently, for each unique pairing of seed regions, we correlated RSFC strength with reading standard scores across individuals in each of the two groups (child, adult) separately, and converted the resultant RSFC-behavior correlations using Fisher's $r$-to- $z$ transformation. We then directly compared the $z$-transformed correlations between the child and adult groups using the aforementioned $Z$-statistic formula.

\section{Results}

Primary analyses

Common RSFC-reading competence relationships

For both age groups, RSFC was positively associated with reading standard scores within functional connections associated with the PCG and IFGop seeds. Peak coordinates of these relationships are reported in Table 3. RSFC between the PCG seed and other motor areas exhibited significant positive correlations with reading competence in each age group: the left postcentral gyrus (L.PstCG) (children, $R^{2}=0.37, p<0.01$; adults, $R^{2}=0.31, p<$ 0.01 ) (Fig. $3 A$ ), the supplementary motor area/posterior cingulate cortex (SMA/PCC) (children, $R^{2}=0.34, p<0.01$; adults, $R^{2}$ $=0.24, p<0.05$ ) (Fig. $3 B$ ), and right postcentral/precentral gyrus (R.PstCG/PCG) (children, $R^{2}=0.27, p<0.01$; adults, $R^{2}$ $=0.42, p<0.001$ ) (Fig. $3 C$ ). Similar positive RSFC-behavior relationships were observed for RSFC between the IFGop seed (Broca's area) and the posterior part of the left superior temporal gyrus (L.STG) (Wernicke's area), extending toward the left middle temporal gyrus (children, $R^{2}=0.20, p<0.05$; adults, $R^{2}=$ $0.31, p<0.01$ ) (Fig. 3D). The magnitude of the RSFC-behavior relationships observed in the two groups only differed for one connection-specifically that between the left PCG seed and right postcentral/precentral gyrus, with the magnitude of the positive relationship being significantly greater in the adult group than in children.

Of note, connections exhibiting RSFC-behavior relationships in both groups tended not to show age group-related differences in connectivity strength (i.e., mean RSFC strength for the two groups did not differ). The one notable exception is the connection between Broca's and Wernicke's areas, where RSFC is significantly greater in adults $(t=2.4, \mathrm{df}=48, p<0.05)$ (Fig. 3D, see scatter plots on the left and right axes).

\section{Divergent $R S F C$-reading competence relationships}

To identify functional connections exhibiting divergent (i.e., age group specific) relationships with reading competence, we performed direct group comparisons of the correlations between RSFC and reading standard scores. We observed significant age grouprelated differences in the relationship between reading competence and RSFC within the functional connections associated with the FFG and IPS seeds. Peak coordinates are reported in Table 3. First, adults exhibited significant positive RSFC-behavior relationships for RSFC between the FFG seed and two phonological regions (Jobard et al., 2003): the pars opercularis of the left IFG (L.IFGop) $\left(r=0.72, R^{2}\right.$ $=0.52, p<0.001$ ) (Fig. $4 A$ ) and the left inferior parietal lobule (L.IPL) $\left(r=0.65, R^{2}=0.42, p<0.001\right)$ (Fig. $\left.4 B\right)$. In children, the direction of these RSFC-behavior relationships was negative (L.IFGop, $r=-0.42, R^{2}=0.18, p<0.05$; L.IPL, $r=-0.25, R^{2}=0.06$,
Table 3. MNI coordinates of peak RSFC-reading competence relationships

\begin{tabular}{|c|c|c|c|c|c|c|c|}
\hline \multirow{2}{*}{$\begin{array}{l}\text { RSFC-behavior } \\
\text { relationships }\end{array}$} & \multirow[b]{2}{*}{ Seed } & \multirow[b]{2}{*}{ Cluster location } & \multirow{2}{*}{$\begin{array}{l}\text { Cluster size } \\
\text { (voxels) }\end{array}$} & \multicolumn{3}{|c|}{ Peak (MNI) } & \multirow[b]{2}{*}{ Peak Z } \\
\hline & & & & $x$ & $y$ & $z$ & \\
\hline \multirow[t]{4}{*}{ Common } & \multirow[t]{3}{*}{ PCG } & L.PstCG & 1783 & -26 & -26 & 66 & 3.61 \\
\hline & & SMA/PCC & 302 & -2 & -14 & 44 & 3.07 \\
\hline & & R.PstCG/PCG & 1394 & 34 & -32 & 64 & 4.40 \\
\hline & IFGop & L.STG/MTG & 206 & -64 & -50 & 8 & 3.15 \\
\hline \multirow[t]{6}{*}{ Divergent } & \multirow[t]{4}{*}{ FFG } & L.IFGop & 1179 & -48 & 12 & 10 & 4.08 \\
\hline & & L.IPL & 249 & -50 & -40 & 38 & 3.47 \\
\hline & & PRC/PCC & 1610 & 10 & -46 & 24 & 4.75 \\
\hline & & VMPFC & 758 & -2 & 44 & -6 & 4.27 \\
\hline & \multirow[t]{2}{*}{ IPS } & L. Thalamus & 615 & -6 & -26 & 6 & 3.42 \\
\hline & & R. Thalamus & 433 & 4 & -16 & 18 & 3.73 \\
\hline
\end{tabular}

L., Left; R., right; PstCG, postcentral gyrus; SMA/PCC, supplementary motor area/posterior cingulate cortex; STG/ MTG, superior temporal gyrus/middle temporal gyrus; IPL, inferior parietal lobule; PRC/PCC, precuneus/posterior cingulate cortex. VMPFC, Ventromedial prefrontal cortex; $Z>2.3, p<0.05$ corrected.

not significant). That is, adults exhibiting stronger positive RSFC between the FFG seed and L.IFGop or L.IPL scored higher on reading competence, while children exhibiting stronger positive RSFC within these same functional connections exhibited lower reading competence.

Second, adults exhibited significant negative relationships between reading competence and RSFC between the FFG seed and two regions of the default network: the precuneus/posterior cingulate cortex (PRC/PCC) $\left(r=-0.67, R^{2}=0.45, p<0.001\right)$ (Fig. $4 C)$ and the ventromedial prefrontal cortex (VMPFC) $(r=$ $-0.56, R^{2}=0.31, p<0.01$ ) (Fig. $4 D$ ). In contrast, children exhibited significant positive correlations between reading competence and RSFC within these same functional connections (PRC/PCC, $r=0.50, R^{2}=0.25, p<0.05$; VMPFC, $r=0.50, R^{2}$ $=0.25, p<0.05)$. These results indicate that adults exhibiting stronger negative RSFC between the FFG seed and default network regions had higher reading competence, while the opposite was true of children. A similar divergent RSFC-behavior relationship was observed for RSFC between the IPS seed and bilateral thalamus (B.THAL) (children, $r=0.65, R^{2}=0.42, p<$ 0.001 ; adults, $r=-0.28, R^{2}=0.08$, not significant) (Fig. $4 E$ ). That is, children exhibiting stronger positive RSFC between the same regions exhibited higher reading competence, while adults exhibiting stronger negative RSFC between the same regions exhibited higher reading competence.

\section{Secondary analyses}

As illustrated in Figure 5, we found significant correlations between ROI-based RSFC strength and reading competence in children and adults, including one that survived Bonferroni correction-between the FFG and IFGop seeds $(Z>2.3, p<$ $0.05)$. Specifically, while adults exhibited a positive relationship between reading competence and FFG-IFGop RSFC, children exhibited the opposite RSFC-behavior relationship. This finding was consistent with the relationship observed in the primary whole-brain analyses.

\section{Discussion}

Our primary aim was to examine similarities and differences in the RSFC-behavior relationships exhibited by typically developing children and adults. Analyses revealed both common (i.e., present in both groups) and divergent (i.e., different between groups) relationships between RSFC and reading competence, which were independent of general intelligence.

\section{Common RSFC-reading competence relationships}

Positive relationships between reading competence and RSFC within functional circuitry were associated with the PCG and IFGop 

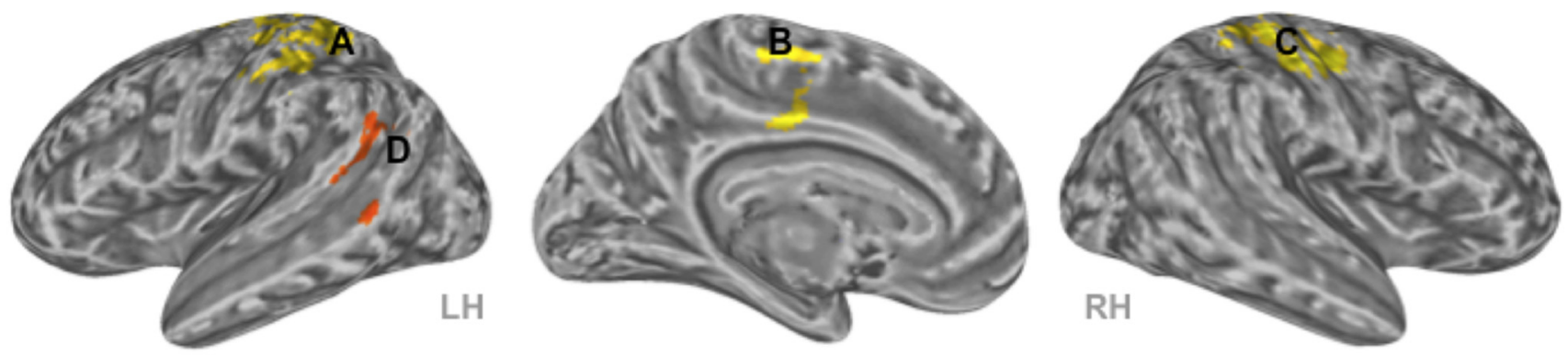

PCG Seed

IFGop Seed

A:L.PCG - L.PstCG

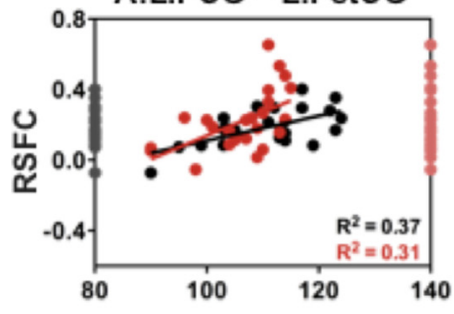

B:L.PCG - SMA

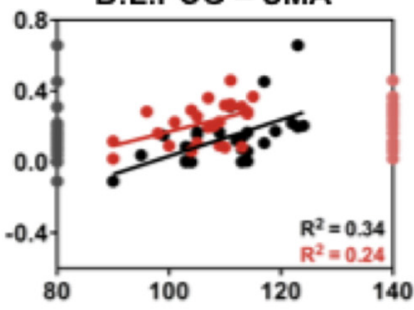

C:L.PCG - R.PstCG

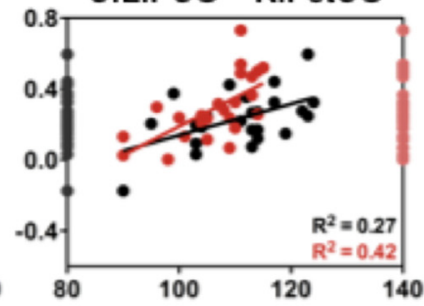

D:L.IFGop - L.STG

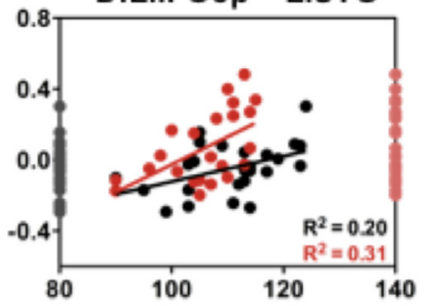

Reading Competence (Standard Scores)

Child OAdult

Figure 3. Age-group common RSFC-reading competence relationships. In both child and adult groups, RSFC of the PCG seed with motor regions (clusters in yellow) and RSFC of the IFGop seed with the posterior part of the left superior temporal gyrus/middle temporal gyrus (a cluster in orange) positively correlated with word reading competence (indexed by reading standard scores). Voxelwise analyses were corrected for multiple comparisons $(Z>2.3, p<0.05$ corrected). Scatter plots $(\boldsymbol{A}-\boldsymbol{D})$ depict RSFC - behavior (reading standard scores) relationships for each age group (children, black circles; adults, red circles). The distribution of RSFC strength for the child and adult groups is displayed on the left and right axes, respectively. L, Left, R, right; PstCG, postcentral gyrus; SMA, supplementary motor area; L.STG, left superior temporal gyrus; LH, left hemisphere; RH, right hemisphere.

seeds in both child and adult groups. The first common RSFCbehavior relationship was observed for RSFC between the PCG seed and other motor areas (i.e., the left postcentral gyrus, the supplementary motor area/posterior cingulate cortex, and the right postcentral/precentral gyrus). This finding is congruent with the motor theory of speech perception (Liberman et al., 1967). The core claim, that speech perception involves access to motor systems, is supported by reports of activation within motor regions during speech sound recognition (Pulvermüller et al., 2006; Meister et al., 2007; Pulvermüller and Fadiga, 2010). Such a close coupling between speech and motor functions also occurs during reading. Longcamp et al. (2005) demonstrated that PCG was responsive to visually presented letters, but not to pseudo-letters (which cannot be sounded out), even in the absence of requiring motor/speech performance. Given the pivotal role of speech perception in the phonological processing underlying reading development (McBride-Chang, 1996; Thomson and Goswami, 2008; Goswami et al., 2011), our findings suggest that strong functional coupling within motor regions serves as a neural framework for efficient reading, regardless of age group, presumably reflecting automatized articulation in response to written words.

The second common RSFC-behavior relationship was observed for a functional connection linking Broca's and Wernicke's areas (Kelly et al., 2010). Specifically, reading competence positively correlated with RSFC between the IFGop seed and left superior temporal gyrus in both age groups. However, the fact that RSFC strength between Broca's and Wernicke's areas was weaker in children than in adults suggests that this connection was still undergoing development in our child participants.
Nonetheless, our RSFC finding suggests that RSFC between Broca's and Wernicke's areas is important in reading competence, regardless of age group. This is in line with the WernickeGeschwind model of language (Geschwind, 1965), which emphasizes "disconnection" of these two regions in individuals with a range of language impairments, including reading disorders (Catani and Mesulam, 2008).

\section{Divergent RSFC-reading competence relationships}

We also observed significant age-group differences in relationships between reading competence and RSFC within functional connections associated with the FFG and IPS seeds. These group differences reflected dissociations in the direction of the RSFCbehavior relationships exhibited by children and adults.

The first divergent relationship was observed for RSFC of the FFG, a region commonly referred to as the visual word form area (Dehaene et al., 2002). This region, located in the ventral visual pathway, is reliably activated by perception of visual forms of words, presumably resulting from extensive experience in visual word recognition during reading (Vinckier et al., 2007; Glezer et al., 2009). Specifically, RSFC-behavior relationships were divergent for RSFC between the FFG and both left inferior frontal gyrus opercularis (Broca's area) and left inferior parietal lobule, areas involved in phonological rehearsal (i.e., articulation) and phonological storage, respectively, during word reading (Paulesu et al., 1993; Stoeckel et al., 2009). While adults exhibited positive relationships between reading competence and RSFC between FFG and these phonology-related regions, children exhibited negative RSFC-behavior relationships. This suggests that stron- 


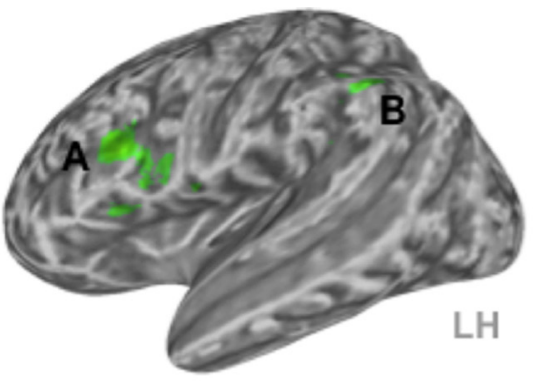

FFG Seed

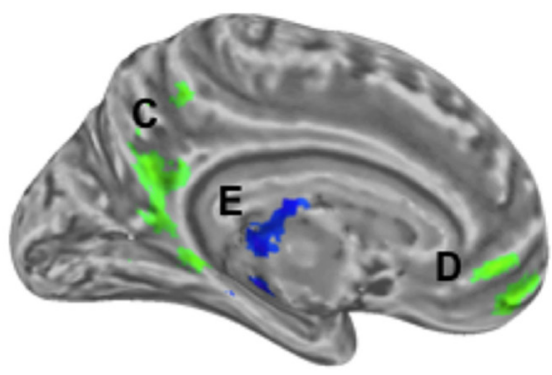

IPS Seed
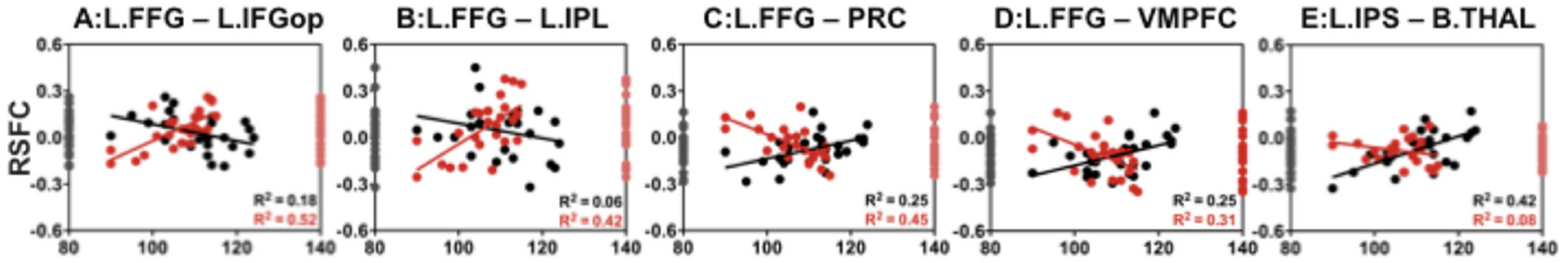

Reading Competence (Standard Scores)

Child

Adult

Figure 4. Age-group divergent RSFC-reading competence relationships. The child and adult groups differed significantly in the relationships between reading competence (indexed by reading standard scores) and RSFC associated with the FFG (clusters in green) and IPS seeds (a cluster in blue). Voxelwise analyses were corrected for multiple comparisons ( $Z>2.3, p<0.05$ corrected). The age-group differences are characterized by dissociations in the direction of the RSFC- behavior relationships. Scatter plots $(\boldsymbol{A}-\boldsymbol{E})$ depict RSFC- behavior (reading standard scores) relationships for each age group (children, black circles; adults, red circles). The distribution of RSFC strength for the child and adult groups is displayed on the left and right axes, respectively. L, Left; B, bilateral; IFGop, inferior frontal gyrus opercularis; IPL, inferior parietal lobule; PRC, precuneus; VMPFC, ventromedial prefrontal cortex; THAL, thalamus; LH, left hemisphere.

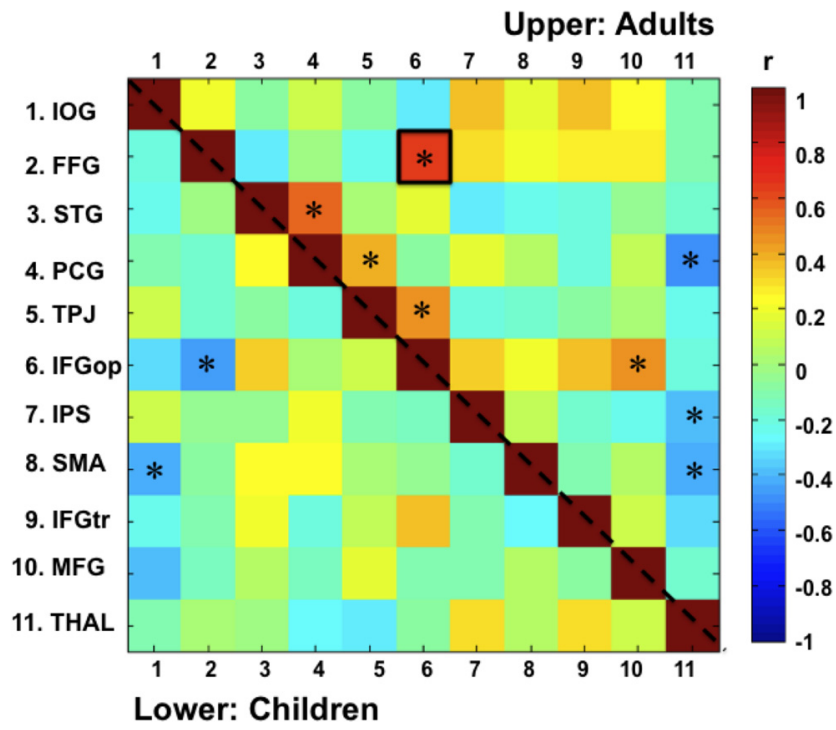

Figure 5. ROI-based analysis of correlations. Significant correlations between RSFC and reading competence were observed in each age group $\left(Z>2.3,{ }^{*} p<0.05\right)$. The correlation in the black square survived Bonferroni correction for multiple comparisons. The bottom and top triangles represent RSFC- behavior relationships in the child and adult groups, respectively. Only RSFC between the fusiform gyrus (2. FFG) and inferior frontal gyrus opercularis (6. IFGop) seeds showed a significant age-group difference in relationships between RSFC and reading competence.

ger positive RSFC between these frontoparietal phonological regions and the FFG is beneficial for reading performance in adults (i.e., the mature brain), but not in children. Such divergent RSF$\mathrm{C}$-behavior relationships may reflect the experience-dependent functional development of FFG, where the magnitude of reading task-induced activation is different between children and adults (Turkeltaub et al., 2008).

A second divergent relationship was observed for RSFC between the FFG and both VMPFC and PRC/PCC. While adults who exhibited the strongest negative RSFC between these regions had the highest reading competence, children who exhibited weaker negative or even positive RSFC between FFG and both $\mathrm{VMPFC}$ and PRC/PCC had higher reading competence. The VMPFC and PRC/PCC constitute two core nodes of the default network, which is typically deactivated during goal-oriented performance (Raichle et al., 2001; Buckner et al., 2008). The RSFCbehavior relationships observed in adults suggest that, in the mature brain, reading competence is associated with increased negative correlation between FFG and default network regions. This is consistent with previous observations that stronger negative correlations between task-active networks and the default network (i.e., functional segregation) are associated with better cognitive performance in adults (Kelly et al., 2008). Our findings suggest that functional segregation between the FFG, a region commonly associated with reading, and default network components indexes efficient reading in the mature brain. In contrast, segregation of the FFG from the default network was not associated with higher reading competence in children. Considering developmental changes in the RSFC of the default network (Fair et al., 2007, 2008; Supekar et al., 2010), this lack of clear functional segregation from the default network may be developmentally typical, and may not be deleterious to reading in children.

A final divergent relationship was observed for the IPS, a core region for top-down control of attention (Corbetta and Shul- 
man, 2002; Fox et al., 2006). RSFC between the IPS seed and bilateral thalamus showed a positive relationship with reading competence in children, but a negative RSFC-behavior relationship in adults. That is, children exhibiting stronger positive RSFC between these regions exhibited higher reading competence, whereas adults exhibiting stronger negative RSFC between the same regions exhibited higher reading competence. The observed strong positive RSFC-behavior correlation in children suggests that children's reading relies on increased connectivity between cortical (IPS) and subcortical (thalamus) attention regions, which does not appear to be necessarily beneficial for adult reading. Although the thalamus is traditionally considered as a relay station passing sensory information to cortical regions (Jones, 1985), recent perspectives highlight the role of the posterior thalamus, the pulvinar, in visual attention (Grieve et al., 2000; Fan et al., 2005). Topographically, the pulvinar largely overlaps with the thalamus cluster we observed (B.THAL) (Fig. 4E). Visual attention has been hypothesized as a "spotlight" for scanning printed letters and words in a serial manner during reading, which is the inevitable and initial step for reading (Vidyasagar, 1999). Accordingly, it can be inferred that functional coupling between corticosubcortical attentional regions is specifically important for children, whose visual scanning during reading has not yet become effortless or automatized. Given that patterns of RSFC between the thalamus and cortical regions undergo developmental changes in childhood and adolescence (Fair et al., 2010), the observed positive RSFC-behavior relationship (lack of segregation) may be developmentally typical and important in children.

Our results should be interpreted in light of limitations. First, the present work does not include older adolescents $(>15$ years old), whose intrinsic functional architecture is likely to be still undergoing dynamic developmental changes (Dosenbach et al., 2010; Power et al., 2010), although their reading competence approaches that of adult levels. Similarly, children younger than 7 years of age were not included, although clearly this period is highly dynamic and critical to the development of reading competence (Brem et al., 2010). It will be of great interest for future work to provide more comprehensive examinations of potential developmental shifts in the neural correlates of reading competence across the lifespan. For young child populations, greater focus on phenotypic measures known to be early precursors of reading competence (e.g., phonological awareness) may prove to be fruitful. Finally, it is worth noting that general intelligence is highly related to reading competence in unimpaired, but not impaired readers (Hulslander et al., 2004; Ferrer et al., 2010). Additionally, other phenotypic variables, such as phonological awareness and working memory (Gathercole et al., 2006), have been associated with reading competence as well. The present work included IQ as a covariate of no interest to remove the potentially confounding effects of general intelligence. Future work would benefit from inclusion of more comprehensive phenotypic information related to reading competence, to account for their potential contributions to the RSFC-reading competence relationships observed in the present work.

In conclusion, we identified both common and divergent relationships between reading competence and RSFC in children and adults. Regardless of age group, stronger coupling among motor regions, as well as between classic language/speech regions, was associated with higher reading competence. In contrast, several RSFC-behavior relationships, particularly those anchored in the left FFG (a region implicated in reading-related visual expertise), diverged between groups. Stronger coupling within FFG-based reading circuitry and stronger functional seg- regation between the FFG and default network components appear to be relevant for efficient and automatized reading in the mature brain (i.e., adults). The absence of these features in children who present age-appropriate reading skills highlights the importance of appreciating developmental changes within neural correlates of reading competence. Our results suggest that RSFC can be used to probe the development of reading competence, and thus may serve to facilitate identifying reading disorders in different age groups.

\section{References}

Adler L, Spencer T (2004) The adult ADHD clinical diagnostic scale (ACDS) V 1.2. New York: New York University School of Medicine.

Andersson J, Jenkinson M, Smith S (2007) Non-linear registration, aka spatial normalisation. In: Technical Report TR07JA2. Oxford, UK: FMRIB.

Biswal B, Yetkin FZ, Haughton VM, Hyde JS (1995) Functional connectivity in the motor cortex of resting human brain using echo-planar MRI. Magnetic resonance in medicine 34:537-541.

Biswal BB, Mennes M, Zuo XN, Gohel S, Kelly C, Smith SM, Beckmann CF, Adelstein JS, Buckner RL, Colcombe S, Dogonowski AM, Ernst M, Fair D, Hampson M, Hoptman MJ, Hyde JS, Kiviniemi VJ, Kötter R, Li SJ, Lin $\mathrm{CP}$, et al (2010) Toward discovery science of human brain function. Proc Natl Acad Sci U S A 107:4734-4739.

Bitan T, Cheon J, Lu D, Burman DD, Gitelman DR, Mesulam MM, Booth JR (2007) Developmental changes in activation and effective connectivity in phonological processing. Neuroimage 38:564-575.

Bolger DJ, Perfetti CA, Schneider W (2005) Cross-cultural effect on the brain revisited: universal structures plus writing system variation. Hum Brain Mapp 25:92-104.

Booth JR, Mehdiratta N, Burman DD, Bitan T (2008) Developmental increases in effective connectivity to brain regions involved in phonological processing during tasks with orthographic demands. Brain Res 1189:78-89.

Brem S, Bach S, Kucian K, Guttorm TK, Martin E, Lyytinen H, Brandeis D, Richardson U (2010) Brain sensitivity to print emerges when children learn letter-speech sound correspondences. Proc Natl Acad Sci U S A 107:7939-7944.

Buckner RL, Andrews-Hanna JR, Schacter DL (2008) The brain's default network. Ann N Y Acad Sci 1124:1-38

Catani M, Mesulam M (2008) The arcuate fasciculus and the disconnection theme in language and aphasia: history and current state. Cortex 44:953-961.

Church JA, Coalson RS, Lugar HM, Petersen SE, Schlaggar BL (2008) A developmental fMRI study of reading and repetition reveals changes in phonological and visual mechanisms over age. Cereb Cortex 18:2054-2065.

Church JA, Petersen SE, Schlaggar BL (2010) The "Task B problem" and other considerations in developmental functional neuroimaging. Hum Brain Mapp 31:852-862.

Corbetta M, Shulman GL (2002) Control of goal-directed and stimulusdriven attention in the brain. Nat Rev Neurosci 3:201-215.

Cox CL, Gotimer K, Roy AK, Castellanos FX, Milham MP, Kelly C (2010) Your resting brain CAREs about your risky behavior. PLoS One 5:e12296.

Cox RW (1996) AFNI: software for analysis and visualization of functional magnetic resonance neuroimages. Comput Biomed Res 29:162-173.

Dehaene S, Le Clec'H G, Poline JB, Le Bihan D, Cohen L (2002) The visual word form area: a prelexical representation of visual words in the fusiform gyrus. Neuroreport 13:321-325.

Di Martino A, Ross K, Uddin LQ, Sklar AB, Castellanos FX, Milham MP (2009) Functional brain correlates of social and nonsocial processes in autism spectrum disorders: an activation likelihood estimation metaanalysis. Biol Psychiatry 65:63-74.

Dosenbach NU, Nardos B, Cohen AL, Fair DA, Power JD, Church JA, Nelson SM, Wig GS, Vogel AC, Lessov-Schlaggar CN, Barnes KA, Dubis JW, Feczko E, Coalson RS, Pruett JR Jr, Barch DM, Petersen SE, Schlaggar BL (2010) Prediction of individual brain maturity using fMRI. Science 329:1358-1361.

Fair DA, Dosenbach NU, Church JA, Cohen AL, Brahmbhatt S, Miezin FM, Barch DM, Raichle ME, Petersen SE, Schlaggar BL (2007) Development of distinct control networks through segregation and integration. Proc Natl Acad Sci U S A 104:13507-13512. 
Fair DA, Cohen AL, Dosenbach NU, Church JA, Miezin FM, Barch DM, Raichle ME, Petersen SE, Schlaggar BL (2008) The maturing architecture of the brain's default network. Proc Natl Acad Sci USA 105:4028-4032.

Fair DA, Cohen AL, Power JD, Dosenbach NU, Church JA, Miezin FM, Schlaggar BL, Petersen SE (2009) Functional brain networks develop from a "local to distributed" organization. PLoS Comput Biol 5:e1000381.

Fair DA, Bathula D, Mills KL, Dias TG, Blythe MS, Zhang D, Snyder AZ, Raichle ME, Stevens AA, Nigg JT, Nagel BJ (2010) Maturing thalamocortical functional connectivity across development. Front Syst Neurosci 4:10.

Fan J, McCandliss BD, Fossella J, Flombaum JI, Posner MI (2005) The activation of attentional networks. Neuroimage 26:471-479.

Ferrer E, Shaywitz BA, Holahan JM, Marchione K, Shaywitz SE (2010) Uncoupling of reading and IQ over time: empirical evidence for a definition of dyslexia. Psychol Sci 21:93-101.

First MB, Williams JBW, Spitzer RL, Gibbon M (2002) Structured Clinical Interview for DSM-IV-TR Axis I Disorders, Research Version, Nonpatient Edition (SCID-I/NP). New York: New York State Psychiatric Institute.

Fleysher R, Fleysher L, Inati S (2005) Fast direct image reconstruction for MRI and fMRI in the presence of field inhomogeneities and T2* decay. In: Proceedings of the 13th Annual Meeting of the International Society for Magnetic Resonance in Medicine, Miami, FL, May.

Fox MD, Corbetta M, Snyder AZ, Vincent JL, Raichle ME (2006) Spontaneous neuronal activity distinguishes human dorsal and ventral attention systems. Proc Natl Acad Sci U S A 103:10046-10051.

Gaillard WD, Balsamo LM, Ibrahim Z, Sachs BC, Xu B (2003) fMRI identifies regional specialization of neural networks for reading in young children. Neurology 60:94-100.

Gathercole SE, Alloway TP, Willis C, Adams AM (2006) Working memory in children with reading disabilities. J Exp Child Psychol 93:265-281.

Geschwind N (1965) Disconnexion syndromes in animals and man. I. Brain $88: 237-294$

Glezer LS, Jiang X, Riesenhuber M (2009) Evidence for highly selective neuronal tuning to whole words in the "visual word form area." Neuron 62: 199-204.

Goswami U, Wang HL, Cruz A, Fosker T, Mead N, Huss M (2011) Language-universal sensory deficits in developmental dyslexia: English, Spanish, and Chinese. J Cogn Neurosci 23:325-337.

Grieve KL, Acuña C, Cudeiro J (2000) The primate pulvinar nuclei: vision and action. Trends Neurosci 23:35-39.

Hampson M, Tokoglu F, Sun Z, Schafer RJ, Skudlarski P, Gore JC, Constable RT (2006) Connectivity-behavior analysis reveals that functional connectivity between left BA39 and Broca's area varies with reading ability. Neuroimage 31:513-519.

Houdé O, Rossi S, Lubin A, Joliot M (2010) Mapping numerical processing, reading, and executive functions in the developing brain: an fMRI metaanalysis of 52 studies including 842 children. Dev Sci 13:876-885.

Hulslander J, Talcott J, Witton C, DeFries J, Pennington B, Wadsworth S, Willcutt E, Olson R (2004) Sensory processing, reading, IQ, and attention. J Exp Child Psychol 88:274-295.

Jenkinson M, Smith S (2001) A global optimisation method for robust affine registration of brain images. Med Image Anal 5:143-156.

Jenkinson M, Bannister P, Brady M, Smith S (2002) Improved optimization for the robust and accurate linear registration and motion correction of brain images. Neuroimage 17:825-841.

Jobard G, Crivello F, Tzourio-Mazoyer N (2003) Evaluation of the dual route theory of reading: a metanalysis of 35 neuroimaging studies. Neuroimage 20:693-712.

Jones EG (1985) The thalamus. New York: Plenum.

Kaufman J, Birmaher B, Brent D, Rao U, Ryan N (1996) The schedule for affective disorders and schizophrenia for school-age children. Pittsburgh: University of Pittsburgh Medical Center.

Kelly AM, Uddin LQ, Biswal BB, Castellanos FX, Milham MP (2008) Competition between functional brain networks mediates behavioral variability. Neuroimage 39:527-537.

Kelly AM, Di Martino A, Uddin LQ, Shehzad Z, Gee DG, Reiss PT, Margulies DS, Castellanos FX, Milham MP (2009) Development of anterior cingu- late functional connectivity from late childhood to early adulthood. Cereb Cortex 19:640-657.

Kelly C, Uddin LQ, Shehzad Z, Margulies DS, Castellanos FX, Milham MP, Petrides M (2010) Broca's region: linking human brain functional connectivity data and non-human primate tracing anatomy studies. Eur J Neurosci 32:383-398.

Koyama MS, Kelly C, Shehzad Z, Penesetti D, Castellanos FX, Milham MP (2010) Reading networks at rest. Cereb Cortex 20:2549-2559.

Liberman AM, Cooper FS, Shankweiler DP, Studdert-Kennedy M (1967) Perception of the speech code. Psychol Rev 74:431-461.

Longcamp M, Anton JL, Roth M, Velay JL (2005) Premotor activations in response to visually presented single letters depend on the hand used to write: a study on left-handers. Neuropsychologia 43:1801-1809.

McBride-Chang C (1996) Models of speech perception and phonological processing in reading. Child Dev 67:1836-1856.

Meister IG, Wilson SM, Deblieck C, Wu AD, Iacoboni M (2007) The essential role of premotor cortex in speech perception. Curr Biol 17:1692-1696.

Paulesu E, Frith CD, Frackowiak RS (1993) The neural correlates of the verbal component of working memory. Nature 362:342-345.

Power JD, Fair DA, Schlaggar BL, Petersen SE (2010) The development of human functional brain networks. Neuron 67:735-748.

Pulvermüller F, Fadiga L (2010) Active perception: sensorimotor circuits as a cortical basis for language. Nat Rev Neurosci 11:351-360.

Pulvermüller F, Huss M, Kherif F, Moscoso del Prado Martin F, Hauk O, Shtyrov Y (2006) Motor cortex maps articulatory features of speech sounds. Proc Natl Acad Sci U S A 103:7865-7870.

Raichle ME, MacLeod AM, Snyder AZ, Powers WJ, Gusnard DA, Shulman GL (2001) A default mode of brain function. Proc Natl Acad Sci U S A 98:676-682.

Schlaggar BL, Church JA (2009) Functional neuroimaging insights into the development of skilled reading. Curr Dir Psychol Sci 18:21-26.

Smith SM, Fox PT, Miller KL, Glahn DC, Fox PM, Mackay CE, Filippini N, Watkins KE, Toro R, Laird AR, Beckmann CF (2009) Correspondence of the brain's functional architecture during activation and rest. Proc Natl Acad Sci U S A 106:13040-13045.

Stoeckel C, Gough PM, Watkins KE, Devlin JT (2009) Supramarginal gyrus involvement in visual word recognition. Cortex 45:1091-1096.

Supekar K, Musen M, Menon V (2009) Development of large-scale functional brain networks in children. PLoS Biol 7:e1000157.

Supekar K, Uddin LQ, Prater K, Amin H, Greicius MD, Menon V (2010) Development of functional and structural connectivity within the default mode network in young children. Neuroimage 52:290-301.

Thomson JM, Goswami U (2008) Rhythmic processing in children with developmental dyslexia: auditory and motor rhythms link to reading and spelling. J Physiol Paris 102:120-129.

Toro R, Fox PT, Paus T (2008) Functional coactivation map of the human brain. Cereb Cortex 18:2553-2559.

Turkeltaub PE, Gareau L, Flowers DL, Zeffiro TA, Eden GF (2003) Development of neural mechanisms for reading. Nat Neurosci 6:767-773.

Turkeltaub PE, Flowers DL, Lyon LG, Eden GF (2008) Development of ventral stream representations for single letters. Ann N Y Acad Sci 1145:13-29.

Vidyasagar TR (1999) A neuronal model of attentional spotlight: parietal guiding the temporal. Brain Res Brain Res Rev 30:66-76.

Vinckier F, Dehaene S, Jobert A, Dubus JP, Sigman M, Cohen L (2007) Hierarchical coding of letter strings in the ventral stream: dissecting the inner organization of the visual word-form system. Neuron 55:143-156.

Wang L, Negreira A, LaViolette P, Bakkour A, Sperling RA, Dickerson BC (2010) Intrinsic interhemispheric hippocampal functional connectivity predicts individual differences in memory performance ability. Hippocampus 20:345-351.

Wechsler D (1999) Wechsler Abbreviated Scale of Intelligence. San Antonio: The Psychological Corporation.

Wechsler D (2001) Wechsler Individual Achievement Test-Second Edition (WIAT-II). San Antonio: The Psychological Corporation.

Zuo XN, Kelly C, Di Martino A, Mennes M, Margulies DS, Bangaru S, Grzadzinski R, Evans AC, Zang YF, Castellanos FX, Milham MP (2010) Growing together and growing apart: regional and sex differences in the lifespan developmental trajectories of functional homotopy. J Neurosci 30:15034-15043. 